\section{Imaging the lymphatic system}

\section{Paolo Zamboni}

Vascular Diseases Center, University of Ferrara, Italy

In the last decade fascinating advanced techniques in lymphatic imaging, new magnetic resonance imaging (MRI) agents created by the development of nano-technologies, as well as gene reported technologies have been developed providing us with new tools to interrogate the function of the lymphatic system. For someone like me, who was born locating and cannulating lymphatic capillaries stained with patent blue in the foot of patients, the novelty in lymphatic diagnostics seems unbelievable. Doppler ultrasound for artery and veins permits blood flow imaging depending upon scattering of flowing erythrocytes, while the acellular lymph escapes clinical interrogation using ultrasound. So different imaging strategies have been developed in the challenging field of the lymphatic system.

Indirect MR lymphangiography can be performed by injection of gadolinium-based contrast dye into the dermal and subcutaneous spaces. For instance indirect MR lymphangiography in the upper and lower extremities of lymphedema patients has excellent resolution and more detailed lymphatic routes visualization as compared to routine lymphoscintigraphy. ${ }^{1-4}$ Moreover, the recent introduction of $3 \mathrm{D}$ isotropic fat- suppressed T1- weighted FSE (VISTA [volumetric isotropic turbo spin-echo acquisition]; Philips Medical Systems) maybe determined a paradigm shift with change of the current gold standard for imaging lymphedema $^{5,6}$ (Figure 1). Fat suppression also permits to overcome the low quality imaging related to the non-homogenous tissue density of the co-existing chronic fibrousedema, or with indurative lipodermatosclerosis when phlebo-lymphedema occurs (Figure 1).

We know that the current gold standard, lymphoscintigraphy, provides routinely information to assess the lymphatic function and anatomy in humans, although with low spatial and temporal resolution respect to the above described innovative MR techniques. Lymphoscintigraphy involves the intradermal administration of radio colloid. Imaging is obtained after hours by the means of the gamma camera. This technique permits to assess the lymphatic function by measuring the time from the injection in distant digits and the appearance of the radionuclide tracer in the major lymph nodes, such as inguinal or iliac..$^{7-9}$

Fluorescence micro-lymphangiography (FML) is a fluorescence technique widely used in animal models of disease, but with scarce usage in humans. Owing to the limited penetration depth of light at visible wavelengths and tissue scattering, only the initial capillaries within the first 100 to 150 microns of tissue depth can be visualized in humans. ${ }^{10}$
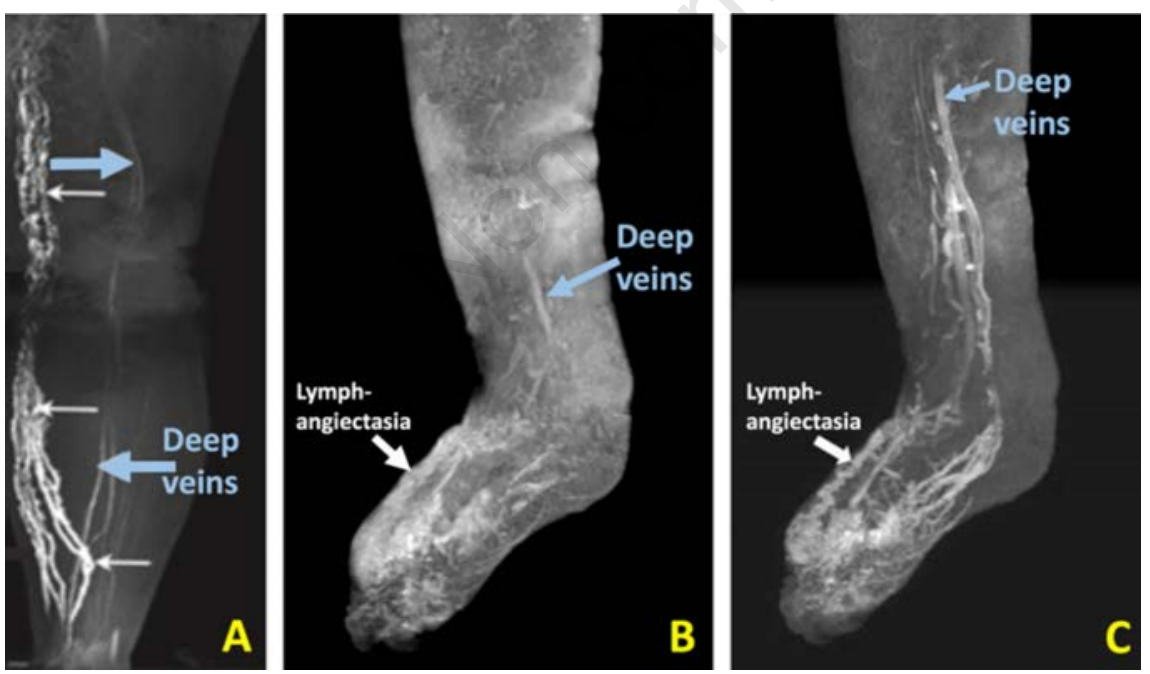

Figure 1. A) Indirect magnetic resonance (MR) lymphangiography in a normal case, with the lymphatic routes of the medial aspect of the lower extremity depicted by the white arrows. B) Indirect MR lymphangiography in a primary lymphedema case with aplasia of lymphatic collectors of the lower limb. The white arrow depicts the ecstasies of the lymphatic capillary of the foot. C) Indirect MR lymphangiography with 3D isotropic fatsuppressed T1- weighted FSE. The improved resolution is well apparent thanks to the suppression of fat sclerosis. Indirect MR lymphangiography permits also to visualize the deep veins as well as to analyze the temporal opacization of both the lymphatic and the venous system. Modified from Jeon et al., 20165 and Sevick-Muraca et al., 2014.6
Correspondence: Paolo Zamboni, Vascular Diseases Center, University of Ferrara, Italy. Tel.: +39.0532.237694.

E-mail: paolozamboni@icloud.com

Received for publication: 14 July 2017. Accepted for publication: 17 July 2017.

This work is licensed under a Creative Commons Attribution 4.0 License (by-nc 4.0).

CCopyright P. Zamboni, 2017

Licensee PAGEPress, Italy

Veins and Lymphatics 2017; 6:6910

doi:10.4081/vl.2017.6910

To improve the use of fluorescence techniques in the human being, NIR fluorescence (NIRF) has been developed. The latter creates the lymphatic imaging, also termed ICG lymphography, through an emerging non-microscopic imaging technology that collects tissue-scattered light to assess conducting and collecting lymphatic vessels at greater penetration depths, but with lower resolution than the initial lymphatic imaged with FML. ${ }^{11}$ In clinical practice could be useful to assess subclinical lymphedema before chronic worsening.

Unlike other lymphatic imaging approaches, NIRF lymphatic images can be collected rapidly with millisecond acquisitions, ${ }^{12}$ allowing for the non-invasive interrogation of function and the quantitative assessment of the lymph-pump frequency and apparent velocity in the collecting and conducting the lymph.

The rapid development and the rapid acquisition of clinical information with the new techniques above described rapidly will change our clinical assessment of lymphedema and related disorders. However, the discrepancy between the advancement of costs and technologies in diagnostics rages against the empiricism of treatment still based on the principles of compression therapy.

\section{References}

1. Lohrmann C, Foeldi E, Langer M. Indirect magnetic resonance lymphangiography in patients with lymphedema preliminary results in humans. Eur J Radiol 2006;59:401-6.

2. Lohrmann C, Foeldi E, Langer M. Diffuse lymphangiomatosis with genital involvement - evaluation with magnetic resonance lymphangiography. Urol Oncol 2011;29:515-22.

3. Lu Q, Delproposto Z, Hu A, et al. MR 
lymphography of lymphatic vessels in lower extremity with gynaecologic oncology-related lymphedema. PLoS One 2012;7:e50319.

4. Notohamiprodjo M, Weiss M, Baumeister RG, et al. MR lymphangiography at $3.0 \mathrm{~T}$ : correlation with lymphoscintigraphy.

Radiology 2012;264:78-87.

5. Jeon JY, Lee SH, Shin MJ, et al. Threedimensional isotropic fast spin-echo MR lymphangiography of T1-weighted and intermediate-weighted pulse sequences in patients with lymphedema. Clin Radiol 2016;71:e56-63.

6. Sevick-Muraca EM, Kwon S,
Rasmussen JC. Emerging lymphatic imaging technologies for mouse and man. J Clin Invest 2014;124:905-14.

7. Battezzati M, Donini I. The use of radioisotopes in the study of the physiopathology of the lymphatic system. J Cardiovasc Surg (Torino) 1964;5:6913.

8. Szuba A, Shin WS, Strauss HW, Rockson S. The third circulation: radionuclide lymphoscintigraphy in the evaluation of lymphedema. J Nucl Med 2003;44:43-57.

9. Zimmerman H, Fessa CK, Rossleigh MA, Wegner EA. Lymphoscintigraphy of lower limb edema. Clin Nucl Med
2012;37:411-5.

10. Modi S, Stanton AW, Mortimer PS, Levick JR. Clinical assessment of human lymph flow using removal rate constants of interstitial macromolecules: a critical review of lymphoscintigraphy. Lymphat Res Biol 2007;5:183-202.

11. Rasmussen JC, Fife CE, Sevick-Muraca EM. Lymphatic imaging in humans with near-infrared fluorescence. Curr Opin Biotechnol 2009;20:74-82.

12. Sevick-Muraca EM. Translation of near-infrared fluorescence imaging technologies: emerging clinical applications. Annu Rev Med 2012;63:217-31. 